\title{
Multiple Lung Hydatid Cysts and Cardiac Hydatid Cyst: A Rare Presentation.
} Garg SM ${ }^{1}$, Umesh ${ }^{2}$, Singh VP', Gupta $N^{4}$, Aggarwal $J^{5}$, Kumar $R^{6}$

\begin{abstract}
:
Hydatid cysts are commonly present in liver. Cardiac hydatid cysts are very rare and represent less than $2 \%$ of all cases of hydatidosis. They can occur as widespread infection or as an isolated event. We present here a case with multiple lung hydatid cysts and a single cardiac hydatid cyst involving the apical antero-interventricular septum. A brief overview of disease and the investigations to establish the diagnosis is discussed here with introduction.
\end{abstract}

Bangladesh Journal of Medical Science Vol. 14 No. 04 October'15. Page: 393-395 DOI: http://dx.doi.org/10.3329/bjms.v14i4.19392

\section{Introduction:}

Hydatid cyst is parasitic infection caused by Echinococcus granulossus and less frequently by Echinococcus multilocularis ${ }^{1}$. It is endemic in India. Cats and dogs are the natural hosts of Echinococcus. Humans are infected by ingesting infected food or water contaminated with eggs or by direct contact with dogs or cats. In humans, the disease most commonly involved the liver and lungs. Cardiac involvement is very rare and occurs in less then $2 \%$ of cases ${ }^{1}$. Cardiac hydatid disease can present with non specific symptoms and therefore imaging plays important role in diagnosis. We report a case of cardiac hydatid cyst and multiple hydatid cysts in lungs.

\section{Patient Presentation:}

A 45 year old male patient reported at MMIMSR, Mullana (Ambala) on 30 ${ }^{\text {th }}$ January, 2013 with complaints of haemoptysis and exertional dyspnoea of 8 months duration. Patient also had high grade fever with chills and rigors 3 days before admission. There was no other significant history except that he had 3 pet dogs in his house. General physical examination was normal. Vital signs were stable. Respiratory sounds were decreased in intensity. Cardiac examination showed no abnormality. Lab investigations revealed mild eosinophilia and mildly deranged liver functions. ECG showed no abnormal finding. Chest radiograph revealed multiple well defined lesions of various sizes and shapes scattered in both lung fields. The cardiac shadow was normal. USG abdomen was normal with no liver involvement. Echocardiography revealed a cystic lesion of $3 \times 3 \mathrm{~cm}$ seen in apical antero-interventricular septum, without significant hemodynamic compromise. CECT chest was done and it revealed multiple well-defined cystic fluid-filled lesions of various sizes and shapes scattered in both lung fields with majority of them showing minimal peripheral capsular enhancement and another cyst of size approximately $2.5 \times 2.5 \mathrm{~cm}$ in apical antero-interventricular septum of heart. The diagnosis was confirmed by the imaging studies without any necessity of histological evaluation. Because both the lungs were diffusely involved with multiple hydatid cysts, surgical intervention was not possible. Patient was treated medically with oral Albendazole $400 \mathrm{mg}$ once daily dose. Patient became asymptomatic within few days of treatment. However, mild breathlessness on exertion persisted. Patient was discharged after one week of hospitalisation.

\section{Discussion:}

Echinococcosis is a tissue infestation; in humans

1. Shyam Garg, Associate Professor, Medicine, (MMIMSR, Mullana (Ambala), Haryana, India

2. Umesh

3. Varinder pal singh

4. Nitin Gupta

5. Jayant Agarwal

6. Ravi Kumar

Resident Medicine, (MMIMSR, Mullana (Ambala), Haryana, India

Corresponds to: Shyam Garg, Associate Professor, Medicine, (MMIMSR,Mullana (Ambala), Haryana, India, Email: drshyamgargmd@yahoo.co.in 


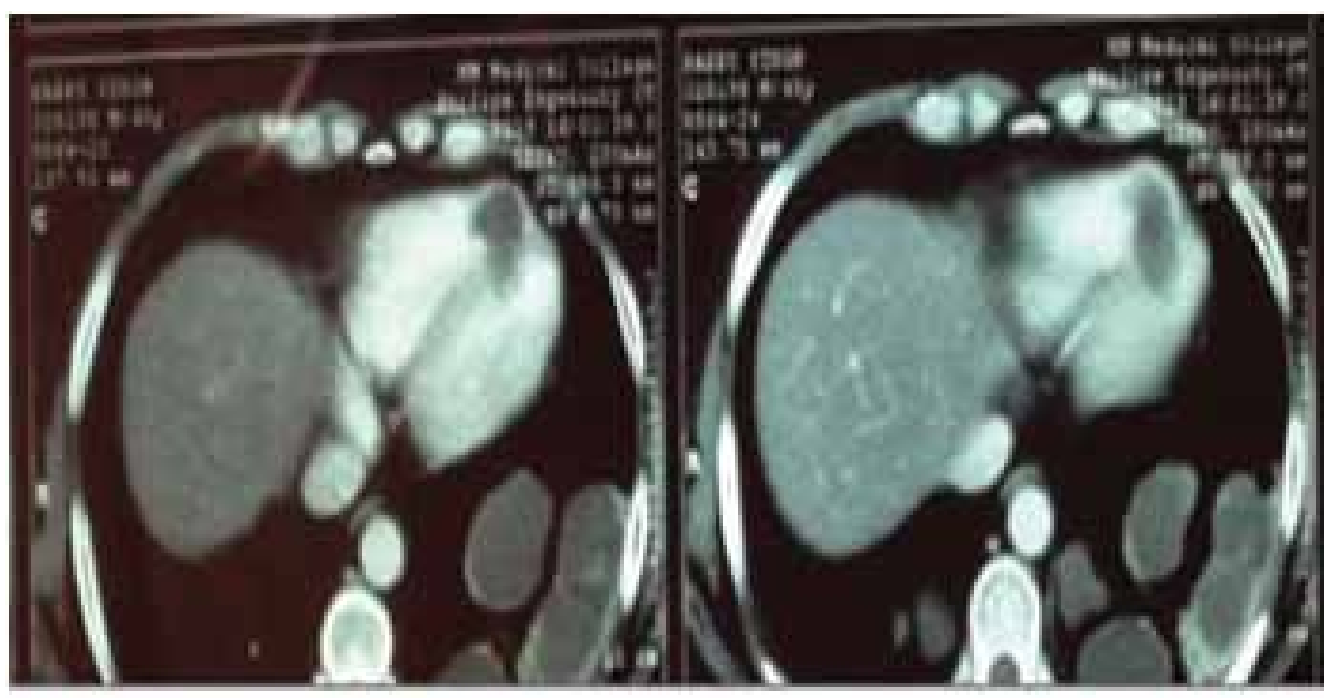

Fig 1: a large cyst in the basal Interventricular septum extending in the right ventricle

it is caused by larva of Echinococcus granulosus. Infection occurs by ingesting infected food or water contaminated with eggs or by direct contact with dogs or cats. The larvae penetrate the gut and are carried by vascular or lymphatic system to liver, lungs and less commonly to other tissues ${ }^{2}$. Our patient had a close contact with dogs for a long time.

Cardiac hydatid cyst disease is a rare $(0.5 \%$ to $2 \%)$, but potentially fatal pathology. It may mimic valvular lesions, give signs of an intracardiac mass, or lead to congestive heart failure ${ }^{3}$. Cardiac hydatid cysts should be suspected in patients who have had contact with dogs and sheep and have signs of cardiac tumour and any mass close to heart on chest $\mathrm{x}-\mathrm{ray}^{4}$. Because the latent phase between infection and presentation of the disease is long and symptoms may be nonspecific,

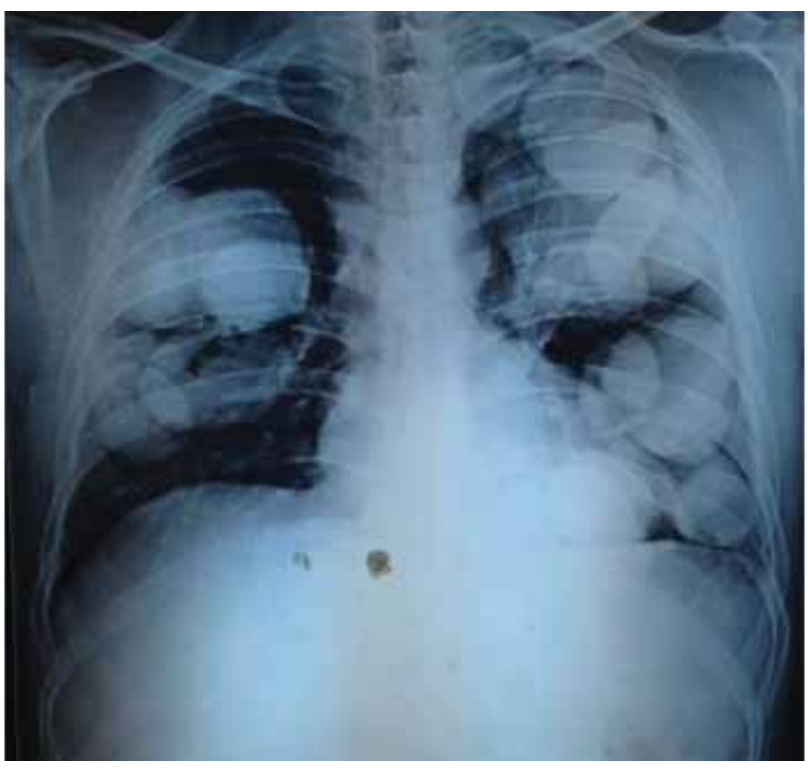

Fig 2: Plain X-Ray chest showing extensive bilateral involvement in lungs and heart (Marked by arrow). establishing an early diagnosis is difficult ${ }^{3}$.

C 1 i n i c a 1 presentation differs according to the number, size, localization, and complication of the cysts, which also makes early diagnosis difficult.

The effects of the cysts on the heart are usually due to cyst perforation, cyst pressure, inducing arrhythmia, angina, valvular dysfunction, pericardial reaction, pulmonary or systemic embolism and anaphylactic reaction. Nearly $90 \%$ of hydatidosis are clinically asymptomatic. . The left ventricle, the part of the heart that has the most abundant blood supply is involved most frequently $(55 \%$ to $60 \%$ ). Involvement of the interventricular septum is reported as $5 \%$ to $9 \%$ of the time 5 .

ELISA, CIEP, bacterial co-agglutination and latex agglutination test are now being used to detect antigen in serum. The serological test are not reliable although it has high specificity but low sensitivity in patients with intact cyst as concentration of antibody in serum is very low until cyst leaks 6 . Recently magnetic resonance imaging has been used to provide

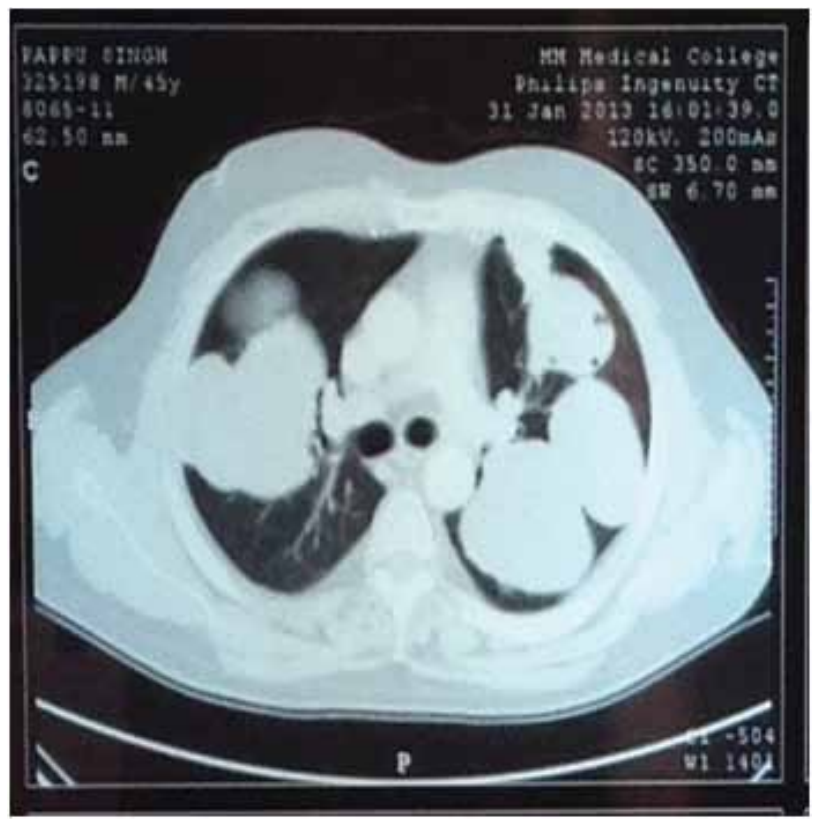

Fig 3: CT showing multiple lung hydatid cysts. 
a diagnosis of hydatid cyst based on low density rim of fibrous tissue rich in pericyst images ${ }^{7}$.

Hydatidosis is still a surgical disease. For simple, accessible cysts, ultrasound or computerised tomography scan guided percutaneous aspiration, instillition of hypertonic saline and reaspiration (PAIR) after 15 mins is the preffered therapy. Alveolar cyst disease may require wide resection (i.e. total lobectomy of liver and lung or even organ transplantation) to remove all cyst material. If cysts are not amenable to resection, oral drug therapy should be started with antihelminthics, either longterm mebendazole ( $40 \mathrm{mg} / \mathrm{kg}$ body weight per day in three divided doses for 6-12 months) or albendazole (400 mg twice daily for 1 to 6 months) for cure and palliation ${ }^{8}$.

\section{References:}

1. Shakibi G, Kokhatri J, Heyat J, Hakaliloo J. Surgical treatment of echinococcal cyst of heart. Report of case and review of literature. J.thorac Cardiovase Surg. 1971;61:755-764

2. Kliegman R. Nelson textbook of pediatrics. Philadelphia: Saunders Elsevier; 2007.

3. Atilgan D, Demirel S, Akkaya V, Korkut F. Left ventricular hydatid cyst: an unusual location of Echinococcus granulosus with multiple organ involvement. $J$ Am Soc Echocardiogr1996;9:212-5. http://dx.doi.org/10.1016/S0894-7317(96)90034-6

4. Altun O, Akalin F, Ayabakan C, Karadag B, Berrak SG, Bilal MS, et al. Cardiac echinococcosis with intra-atrial localization. Turk J Pe-diatr. 2006;48(1):76-9.
5. Kulan K, Tuncer C, Kulan C, et al. Hydatid cyst of the interventricular septum and contribution of magnetic resonance imaging. Acta Cardiol 1995;50:477- 81 .

6. Onurjal E, Elamaci T, Tireli E et al. Surgical treatment of cardiac echinococcosis: report of eight cases. Surgery today 2001;31:325-330. http://dx.doi.org/10.1007/s005950170153

7. Kotoulas G, Magoufis G, Gouliamos A et al. Evaluation of hydatid disease of heart with magnetic resonance imaging. Cardiovasc Intervent Radiol 1996;19:187-189. http://dx.doi.org/10.1007/BF02577618

8. Cecil textbook of Medicine 24th edition. 